\title{
Development of a risk-stratification scoring system for predicting lymphovascular invasion in breast cancer
}

Ma-yi-di-li Ni-jia-ti, Di-li-a-re-mu Ai-hai-ti, Ai-si-ka-er-jiang Huo-jia, Pa-li-dan-mu Wu-mai-er, A-bu-du-ke-you-mu-jiang A-bu-li-zi, Yu Shi, Nu-er-a-mi-na Rou-zi, Wen-jing Su, Guo-zhao Dai and Mai-he-mi-ti-jiang Da-mo-la*

\begin{abstract}
Background: Lymphovascular invasion (LVI) is a vital risk factor for prognosis across cancers. We aimed to develop a scoring system for stratifying LVI risk in patients with breast cancer.

Methods: A total of 301 consecutive patients (mean age, $49.8 \pm 11.0$ years; range, 29-86 years) with breast cancer confirmed by pathological reports were retrospectively evaluated at the authors' institution between June 2015 and October 2018. All patients underwent contrast-enhanced Magnetic Resonance Imaging (MRI) examinations before surgery. MRI findings and histopathologic characteristics of tumors were collected for analysis. Breast LVI was confirmed by postoperative pathology. We used a stepwise logistic regression to select variables and two cutpoints were determined to create a three-tier risk-stratification scoring system. The patients were classified as having low, moderate and high probability of LVI. The area under the receiver operating characteristic curve (AUC) was used to evaluate the discrimination ability of the scoring system.

Results: Tumor margins, lobulation sign, diffusion-weighted imaging appearance, MRI-reported axillary lymph node metastasis, time to signal intensity curve pattern, and HER-2 were selected as predictors for LVI in the point-based scoring system. Patients were considered at low risk if the score was $<3.5$, moderate risk if the score was 3.5 to 6.0 , and high risk if the score was $\geq 6.0$. LVI risk was segmented from 0 to $100.0 \%$ and was positively associated with an increase in risk scores. The AUC of the scoring system was 0.824 (95\% confidence interval [Cl]: 0.776--0.872).
\end{abstract}

Conclusion: This study shows that a simple and reliable score-based risk-stratification system can be practically used in stratifying the risk of LVI in breast cancer.

Keywords: Breast cancer, Magnetic resonance imaging, Lymphovascular invasion, Risk stratification

\section{Background}

Identification of clinically predictive and prognostic factors is considered as an important issue in treatment evaluation of breast cancer. The first study on the prognostic significance of peritumoral lymphovascular invasion (LVI) in breast cancer was reported in 1964 [1], and not a few subsequent studies have reported the association between LVI and clinical outcomes of breast cancer. Accumulating evidence has showed that LVI has an

\footnotetext{
*Correspondence: maihe313@126.com

Department of Radiology, The first people's Hospital of Kashi area, No.120,

Yingbin avenue, Kashi, Xinjiang Uygur Autonomous Region, People's

Republic of China
}

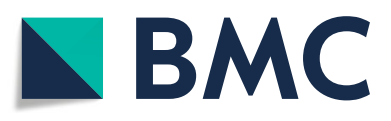

(อ) The Author(s). 2020 Open Access This article is distributed under the terms of the Creative Commons Attribution 4.0 International License (http://creativecommons.org/licenses/by/4.0/), which permits unrestricted use, distribution, and

reproduction in any medium, provided you give appropriate credit to the original author(s) and the source, provide a link to the Creative Commons license, and indicate if changes were made. The Creative Commons Public Domain Dedication waiver (http://creativecommons.org/publicdomain/zero/1.0/) applies to the data made available in this article, unless otherwise stated.

unfavorable prognostic effect on breast cancer survival and recurrence across all molecular subtypes [2], and LVI is regarded as one of the crucial steps in breast cancer metastasis [3-5]. To date, breast cancer LVI is only available at pathological analysis. Preoperative knowledge of LVI can provide valuable information for determining the need for adjuvant chemotherapy or not.

Recently, a preliminary study used nomogram to predict the presence of LVI based on multiparametric magnetic resonance imaging (MRI) and pathological reports [6]. However, using a nomogram in clinical practice might be time-consuming and at risk of difficult interpretation. Klingen TA et al. reported that LVI was 
associated with HER2-positive and several features of aggressive breast cancer such as larger tumor size, higher histological grade, lymph node positive tumors and higher Ki67 expression [7]. Conventional MRI provides morphologic characteristics of the lesions with high spatial resolution, such as the margin morphology, or the internal architecture of the tumors [8]. Functional MRI such as diffusion-weighted imaging (DWI) in a fast time acquisition and without contrast medium gives information about cellularity of breast cancer [9]. Macchini $\mathrm{M}$ et al. found a correlation between dynamic contrast-enhanced (DCE) MR features with LVI [10]. Komatsu S et al. reported that a higher frequency of LVI was observed in the breast cancer lesions with the malignant pattern of time to signal intensity curve (TIC) [11]. This study aimed to develop a scoring system for stratifying the LVI risk of breast cancer based on pretreatment clinical, MRI and pathologic parameters.

\section{Methods}

\section{Basic characteristics of patients}

This retrospective study was approved by the Ethics Committee of the first people's Hospital of Kashi area, and the patient's informed consent was waived. We reviewed a total of 301 consecutive female patients (mean age, $49.8 \pm 11.0$ years; range, $29-86$ years) with biopsy-proven solitary breast cancer admitted to the radiology department between June 2015 and October 2018. All patients underwent a $1.5 \mathrm{~T}$ MRI scan before surgery. Patients with complete clinicopathological characteristics. All patients had received no neoadjuvant therapy prior to operation. Patients with previouslydiagnosed breast cancer or incomplete clinical records were excluded.

Clinicopathological features were collected including age, ER, PR, HER-2, Ki-67 index and histological subtypes. Positive ER or PR was recorded by immunohistochemistry when at least $1 \%$ of the tumor cell nuclei showed staining for ER or PR, respectively [12]. HER-2 was scored as $0,1+, 2+$ or $3+$ in line with the American Society of Clinical Oncology/College of American Pathologists clinical practice guidelines, also adopted by the Italian Society of Pathological Anatomy and Diagnostic Cytology-Italian Division of the International Academy of Pathology (SIAPEC-IAP) [13]: 0, no staining observed or membrane staining that is incomplete or faint/barely perceptible in $\leq 10 \%$ of tumor cells; $1+$, incomplete membrane staining that is faint/barely perceptible within $>10 \%$ of tumor cells; $2+$, circumferential membrane staining that is incomplete and/or weak/ moderate within $>10 \%$ of tumor cells, or complete and circumferential membrane staining that is intense within $\leq 10 \%$ of tumor cells; and $3+$, circumferential membrane staining that is complete and intense within $>10 \%$ of tumor cells. The Ki-67 expression is defined as the percentage of positively stained tumor cells among the total number of malignant cells assessed [14]. The histological subtype of cancer was defined according to the World Health Organization classification.

\section{MR image acquisition}

Preoperative breast MR imaging is a routine practice in our Institution. The MR imaging was performed in the prone position using a dedicated four-channel doublebreast coil with a $1.5 \mathrm{~T}$ system (Siemens Avanto, Germany). Bilateral whole-breast MR imaging was performed using the following sequences and parameters: axial 3D gradient echo (GRE) Dixon T1-weighted sequence $(\mathrm{TR} / \mathrm{TE}=6.86 \mathrm{~ms} / 2.39 \mathrm{~ms}$, section thickness $=$ $2 \mathrm{~mm}$, number of slices $=80, \mathrm{NEX}=1$, matrix $=384 \times$ $384, \mathrm{FOV}=360 \times 360 \mathrm{~mm}$, acquisition time $=2 \mathrm{~min} 19 \mathrm{~s}$ ). Axial T2-weighted short tau inversion recovery (STIR) $(\mathrm{TR} / \mathrm{TI}=2550 \mathrm{~ms} / 170 \mathrm{~ms}, \mathrm{TE}=107 \mathrm{~ms}$, section thickness $=5 \mathrm{~mm}$, number of slices $=25, \mathrm{NEX}=4$, matrix $=$ $320 \times 320, \quad F O V=350 \times 350 \mathrm{~mm}, \quad$ acquisition time $=2$ min $33 \mathrm{~s})$. Axial DWI was obtained with SE sequence $(\mathrm{TR} / \mathrm{TE}=6800 \mathrm{~ms} / 115 \mathrm{~ms}$, section thickness $=5 \mathrm{~mm}$, gradient directions $=3$, matrix $=120 \times 75, F O V=350 \times$ $350 \mathrm{~mm}$, EPI factor $=107$, bandwidth $=758$, $\mathrm{b}$ values $=0$, $800 \mathrm{~s} / \mathrm{mm}^{2}$ ). Axial dynamic contrast-enhanced (DCE) MRI was performed using the volumetric interpolated breath-hold examination (VIBE) fat-suppression sequence $\left(\mathrm{TR} / \mathrm{TE}=3.0 \mathrm{~ms} / 1.42 \mathrm{~ms}\right.$, flip angle $=10^{\circ}$, matrix $=100 \times 80, \quad F O V=150 \mathrm{~mm} \times 120 \mathrm{~mm} \times 70 \mathrm{~mm}$ ), 55 dynamic phases, $7.3 \mathrm{~s}$ per phase, a total of $405 \mathrm{~s}$. At the second phase of DCE scan, a bolus of $0.1 \mathrm{mmol} \mathrm{kg}^{-1}$ of gadodiamide (Omniscan, GE Healthcare) was injected into the antecubital vein at a rate of $3 \mathrm{~mL} / \mathrm{s}$, followed by a $30 \mathrm{~mL}$ saline flush. After the DCE scan, axial and coronal T1-weighted fat-suppression contrast-enhancement sequences were obtained $(\mathrm{TR} / \mathrm{TE}=6.88 \mathrm{~ms} / 2.39 \mathrm{~ms}$, section thickness $=2 \mathrm{~mm}, \quad$ matrix $=384 \times 384, \quad F O V=$ $360 \times 360 \mathrm{~mm}$; TR/TE $=4.85 \mathrm{~ms} / 2.34 \mathrm{~ms}$, section thickness $=3 \mathrm{~mm}$, matrix $=320 \times 320, \mathrm{FOV}=380 \times 380 \mathrm{~mm}$ ).

\section{MR image analyses}

MR image analyses were performed on the picture archiving and communication system and Siemens postprocessing workstation. Two radiologists with 10 and 20 years of experience with breast imaging, respectively, who were blinded to the pathological information, reviewed all MR images independently. If different assessments were assigned by the two radiologists, discrepancies were discussed to reach a consensus, and consensus was achieved between the two radiologists for all cases. MR image analyses included tumor location (upper-outer quadrant, upper-inner quadrant, lowerouter quadrant, lower-inner quadrant, or central 
position), tumor size (maximum diameter), margins (well- defined or ill- defined), lobulation sign (absence or presence), spiculation sign (absence or presence), MRIreported axillary lymph node metastasis (ALNM) (absent, single or multiple), DWI appearance (slight/moderate hyperintensity or marked hyperintensity), timeintensity curve (TIC) patterns (type I, a straight or curved line; type II, a sharp bend after the initial upslope with plateau thereafter; type III, contrast washout was evident after an initial upslope). A $3 \times 3$ pixel standardsized regions of interest ( $\mathrm{ROI}$ ) was selected within the areas of obvious intense enhancement in the tumor. TIC was generated. Metastatic axillary lymph nodes were nodes with major axis $>10 \mathrm{~mm}$ and hilus of lymph node disappeared.

\section{Reference standard}

LVI was evaluated on routine hematoxylin-eosin-stained sections according to the method of Pinder et al. [15]. LVI was present if tumor cells were observed in the space of endothelial cells. Two pathologists with more than 10 years of experience reviewed all pathological images independently.

\section{Point-based scoring system development}

We used a stepwise logistic regression to select variables. Variables in the stepwise analysis with $p$ values $<0.05$ were considered significant. For each significant variable a regression coefficient was obtained. Factors significantly associated with breast LVI in univariable and multivariable logistic regression analysis using stepwise method are listed in Table 1. Results of the univariable and multivariable logistic regression analysis were shown as odds ratio (OR) and corresponding 95\% CI. Points for the prediction rule were assigned by doubling the value of the regression coefficients from the stepwise logistic regression and rounding to the nearest 0.5 . In an effort to simplify eventual clinical application, a point-based scoring system was constructed using the method described by Sullivan et al. [16]. We then created cut points to classify patients as having low, moderate and high risk of LVI with optimum LVI rate for each category. To estimate the discriminating power of the scoring system, receiver operator characteristic (ROC) curves were created and the areas under the curves (AUCs) were calculated. The LVI rate in each category, according to pathology also determined, along with $95 \%$

Table 1 Factors significantly associated with breast LVI in univariable and multivariable logistic regression analysis using stepwise method

\begin{tabular}{|c|c|c|c|c|}
\hline \multirow[t]{2}{*}{ Variable } & \multicolumn{2}{|c|}{ Univariable regression } & \multicolumn{2}{|c|}{ Multivariable regression } \\
\hline & Odds Ratio $(95 \% \mathrm{Cl})$ & $\overline{p \text {-value }}$ & Odds Ratio $(95 \% \mathrm{Cl})$ & $p$-value \\
\hline \multicolumn{5}{|l|}{ Margins } \\
\hline Well-defined & reference & & reference & \\
\hline IIl-defined & $4.99(2.69-9.90)$ & $<0.001$ & $3.46(1.69-7.49)$ & 0.001 \\
\hline \multicolumn{5}{|l|}{ Lobulation sign } \\
\hline Absence & reference & & reference & \\
\hline Presence & $4.00(2.38-6.89)$ & $<0.001$ & $3.66(1.90-7.30)$ & $<0.001$ \\
\hline \multicolumn{5}{|l|}{ DWI appearance } \\
\hline Slight hyperintensity & reference & & reference & \\
\hline Marked hyperintensity & $3.46(2.02-6.15)$ & $<0.001$ & $2.67(1.42-5.17)$ & 0.003 \\
\hline \multicolumn{5}{|l|}{ MRI-reported ALNM } \\
\hline Absence & reference & & reference & \\
\hline Single & $0.52(0.26-1.04)$ & 0.068 & $0.33(0.14-0.75)$ & 0.014 \\
\hline Multiple & $0.26(0.15-0.45)$ & $<0.001$ & $0.26(0.13-0.51)$ & $<0.001$ \\
\hline \multicolumn{5}{|l|}{ TIC type } \\
\hline 1 & reference & & reference & \\
\hline$\|$ & $12.16(2.50-219.37)$ & 0.015 & 10.55 (1.87-201.14) & 0.032 \\
\hline III & $29.71(5.55-553.11)$ & 0.001 & $26.00(4.07-520.41)$ & 0.004 \\
\hline \multicolumn{5}{|l|}{ HER2 } \\
\hline- & reference & & reference & \\
\hline+ & $1.90(0.61-7.24)$ & 0.298 & $1.24(0.31-5.89)$ & 0.739 \\
\hline $2+$ & $2.76(0.98-9.89)$ & 0.078 & $2.48(0.70-10.92)$ & 0.195 \\
\hline $3+$ & $3.32(1.11-12.41)$ & 0.046 & $6.37(1.64-30.54)$ & 0.017 \\
\hline
\end{tabular}


Table 2 Comparison of clinicopathologic and radiological features between patients with and without LVI

\begin{tabular}{|c|c|c|c|}
\hline Parameters & No. of patients without LVI $(n=197)$ & No. of patients with LVI $(n=104)$ & $P$ value \\
\hline \multicolumn{4}{|l|}{ Age (years) } \\
\hline Mean & $50.0 \pm 10.8$ & $49.6 \pm 11.4$ & 0.767 \\
\hline Median & 49.0 & 48.0 & - \\
\hline$<50(n=167)$ & $105(62.9)$ & $62(37.1)$ & 0.294 \\
\hline$\geq 50(n=134)$ & $92(68.7)$ & $42(31.3)$ & \\
\hline \multicolumn{4}{|l|}{ Tumor location } \\
\hline Upper-outer quadrant ( $n=14$ 6) & $96(65.8)$ & $50(34.2)$ & 0.028 \\
\hline Lower-outer quadrant $(n=44 \quad)$ & $33(75.0)$ & $11(25.0)$ & \\
\hline Upper-inner quadrant $(n=74 \quad)$ & $51(68.9)$ & $23(31.1)$ & \\
\hline Lower-inner quadrant $(n=19 \quad)$ quadrant $(n=31)$ & $11(57.9)$ & $8(42.1)$ & \\
\hline Central position ( $n=18)$ & $6(33.3)$ & $12(66.7)$ & \\
\hline \multicolumn{4}{|l|}{ Tumor size $(\mathrm{mm})$} \\
\hline$<30(n=176)$ & $124(70.5)$ & $52(29.5)$ & 0.030 \\
\hline$\geq 30(n=125)$ & $73(58.4)$ & $52(41.6)$ & \\
\hline \multicolumn{4}{|l|}{ Margins } \\
\hline Well-defined $(n=95)$ & $82(86.3)$ & $13(13.7)$ & $<0.001$ \\
\hline III-defined $(n=206)$ & $115(55.8)$ & $91(44.2)$ & \\
\hline \multicolumn{4}{|l|}{ Lobulation sign Hyperechogenicity $(n=61)$} \\
\hline Absence $(n=135)$ & $110(81.5)$ & $25(18.5)$ & $<0.001$ \\
\hline Presence $(n=166)$ & $87(52.4)$ & $79(47.6)$ & \\
\hline \multicolumn{4}{|l|}{ Spiculation sign } \\
\hline Absence $(n=122)$ & $88(72.1)$ & $34(27.9)$ & 0.044 \\
\hline Presence $(n=179)$ & $109(60.9)$ & $70(39.1)$ & \\
\hline \multicolumn{4}{|l|}{ MRI-reported ALNM } \\
\hline Absent $(n=92)$ & $43(46.7)$ & $49(53.3)$ & $<0.001$ \\
\hline Single $(n=51)$ & $32(62.7)$ & $19(37.3)$ & \\
\hline Multiple $(n=158)$ & $122(77.2)$ & $36(22.8)$ & \\
\hline \multicolumn{4}{|l|}{ DWI appearance } \\
\hline Slight-moderate hyperintensity $(n=113)$ & $92(81.4)$ & $21(18.6)$ & $<0.001$ \\
\hline Marked hyperintensity $(n=188)$ & $105(55.9)$ & $83(44.1)$ & \\
\hline \multicolumn{4}{|l|}{ TIC pattern } \\
\hline Type I $(n=25)$ & $24(96.0)$ & $1(4.0)$ & $<0.001$ \\
\hline Type II $(n=229)$ & $152(66.4)$ & 77 (33.6) & \\
\hline Type III $(n=47)$ & $21(44.7)$ & $26(55.3)$ & \\
\hline \multicolumn{4}{|l|}{$E R(\%)$} \\
\hline$<50(n=116)$ & $72(62.1)$ & $44(37.9)$ & 0.329 \\
\hline$\geq 50(n=185)$ & $125(67.6)$ & $60(32.4)$ & \\
\hline \multicolumn{4}{|l|}{ PR (\%) } \\
\hline$<50(n=191)$ & $120(62.8)$ & $71(37.2)$ & 0.208 \\
\hline$\geq 50(n=110)$ & $77(70.0)$ & $33(30.0)$ & \\
\hline \multicolumn{4}{|l|}{ HER2 } \\
\hline$-(n=23)$ & $19(82.6)$ & $4(17.4)$ & 0.132 \\
\hline$+(n=63)$ & $45(71.4)$ & 18 (28.6) & \\
\hline$++(n=149)$ & $93(63.3)$ & $54(36.7)$ & \\
\hline
\end{tabular}


Table 2 Comparison of clinicopathologic and radiological features between patients with and without LVI (Continued)

\begin{tabular}{|c|c|c|c|}
\hline Parameters & No. of patients without LVI $(n=197)$ & No. of patients with LVI $(n=104)$ & $P$ value* \\
\hline$+++(n=68)$ & $40(58.8)$ & $28(41.2)$ & \\
\hline \multicolumn{4}{|l|}{ Ki-67 (\%) index } \\
\hline$<20(n=99)$ & $64(64.6)$ & $35(35.4)$ & \multirow[t]{2}{*}{0.838} \\
\hline$\geq 20(n=202)$ & $133(65.8)$ & $69(34.2)$ & \\
\hline \multicolumn{4}{|l|}{ Histological type } \\
\hline IDC grade $3(n=106)$ & $58(54.7)$ & $48(45.3)$ & \multirow[t]{3}{*}{$<0.001$} \\
\hline IDC grade $2(n=149)$ & $98(65.8)$ & $51(34.2)$ & \\
\hline DCIS $(n=46)$ & $41(89.0)$ & $5(11.0)$ & \\
\hline
\end{tabular}

Note: unless otherwise indicated, data are numbers of nodules, and numbers in parentheses are percentages. ${ }^{*} P$ value were calculated by using generalized estimating equation analysis. DWI diffusion-weighted imaging, $T / C$ time-intensity curve, $E R$ estrogen receptor, $P R$ progesterone receptor, $H E R 2$ human epidermal growth factor receptor, IDC invasive ductal carcinoma. DCIS ductal carcinoma in situ

confidence intervals (CIs). Specifically, we showed the use of point-based scoring system in the result section by taking two cases for example, one with LVI and another without LVI.

\section{Statistical analysis}

$\mathrm{R}$ version 3.2.3 was used for statistical analysis. As for continuous variables, data were expressed as mean \pm standard deviation (SD), while for categorial variables, data were expressed as counts and percentages (n, \%). Continuous and categorial variables were compared by independent $\mathrm{t}$ tests, Mann-whitney $\mathrm{U}$ test, or Chisquare, if appropriate. The AUCs of logistic regression model and scoring system were compared with Delong test. A $p<0.05$ were considered significant.

\section{Results}

\section{Patient and tumor characteristics}

Among the 301 lesions, 106 (35.2\%) were invasive ductal carcinoma (IDC) (histological grade 3), 149 (49.5\%) were IDC (histological grade 2), 46 (15.3\%) were ductal carcinoma in situ (DCIS). In total, 104 (34.6\%) patients (mean age, $49.6 \pm 11.4$ ) had LVI and 197 (65.4\%) patients (mean age, $49.6 \pm 11.4$ ) without LVI. Comparison of clinicopathologic and radiological features between patients with and without LVI is shown in Table 2.

\section{Development of point-based scoring system for predicting LVI}

By multivariate logistic regression analysis, seven variables were significantly related to LVI and were assigned scores for the final prediction rule: margins (ill-defined [1.0 points], lobulation sign (presence [1.5 points]), DWI appearance (marked hyperintensity [1.0 points]), TIC pattern (type II [2.5 points], type III [3.0 points]), MRIreported ALNM (single [-1.0 points], multiple [-1.5 points $]$ ), HER2 (+ [0 points $], 2+[1.0$ points $], 3+[1.5$ points]) (Table 3 ). If patients had $<3.5$ points the probability of LVI was low with $5.3 \%$ (95\% CI: $0.1-1.0 \%$ ) having LVI. A score of 3.5-6.0 was moderate probability with $28.9 \%$ (95\% CI: 21.7-36.2\%) having LVI; a score of $\geq 6.0$ was high probability with $76.7 \%$ (95\% CI: $66.8-$ 86.6\%) having LVI.

\section{The performance of point-based scoring system for predicting LVI}

The logistic regression model obtained an AUC of 0.830 (95\%CI: 0.783-0.0.878). The proposed scoring system

Table 3 Points assigned to significant variables to determine patient score

\begin{tabular}{ll}
\hline Variables & Points \\
\hline Margins & 0 \\
Well-defined & 1.0 \\
ill-defined & \\
Lobulation sign & 0 \\
absence & 0.5 \\
presence & \\
DWI appearance & 0 \\
slight-moderate hyperintensity & 1.0 \\
marked hyperintensity & \\
TIC pattern & 0 \\
type I & 2.5 \\
type II & 3.0 \\
type III & \\
MRI-reported ALNM & \\
absent & \\
single & \\
multiple & \\
HER2 & -1.0 \\
- & \\
2+ & \\
\hline+ & 0 \\
\hline
\end{tabular}


achieved an AUC of 0.824 (95\%CI: 0.776-0.872). There were no significant AUC differences between the logistic regression model and the scoring system $(p=0.239)$ (Fig. 1). Table 4 presents the risk of LVI according to the point based scoring risk stratification system. LVI risk ranged from 0 to $100.0 \%$ and increased as the risk score increased and peaked at $100.0 \%$ in the scoring risk stratification model.

\section{Examples of the risk-stratification scoring system in use}

For example, patient 1 aged 59 years, who had a tumor in her right breast, location in the.

upper-outer quadrant, with maximum diameter of $19 \mathrm{~mm}$, well-defined margins, absence of lobulation sign, marked hyperintensity on DWI images, type I TIC, and no ALNM (Fig. 2a-f). Biopsy results showed a IDC (grade 2) and positive HER-2 (+). The risk score of LVI assessed by our system was 1 . Pathology report showed that the tumor was free of LVI. Patient 2 aged 57 years, who had a tumor in her left breast, location in the upper-inner quadrant, with maximum diameter of $35 \mathrm{~mm}$, ill-defined margins, presence of lobulation sign, marked hyperintensity on DWI images, type II TIC, and multiple ALNM (Fig. 2g-l). Biopsy results showed a IDC (grade 3 ) and positive HER-2 (3+). The risk score of LVI could be

Table 4 Lymphovascular invasion risk according to the pointbased scoring system

\begin{tabular}{llll}
\hline Risk score & LVI risk (\%) & Total number & Number of LVI \\
\hline-1.5 & $0 \%$ & 1 & 0 \\
-0.5 & $0 \%$ & 2 & 0 \\
0 & $0 \%$ & 1 & 0 \\
1 & $0 \%$ & 1 & 0 \\
1.5 & $0 \%$ & 12 & 0 \\
2 & $0 \%$ & 4 & 0 \\
2.5 & $0 \%$ & 14 & 0 \\
3 & $3.8 \%$ & 15 & 3 \\
3.5 & $19.4 \%$ & 26 & 1 \\
4 & $26.1 \%$ & 31 & 6 \\
4.5 & $28.2 \%$ & 23 & 6 \\
5 & $35.9 \%$ & 39 & 11 \\
5.5 & $35 \%$ & 39 & 14 \\
6 & $62.9 \%$ & 20 & 7 \\
6.5 & $75 \%$ & 35 & 22 \\
7 & $92.9 \%$ & 12 & 9 \\
7.5 & $100 \%$ & 10 & 13 \\
8 & $100 \%$ & 2 & 10 \\
Total & $34.6 \%$ & 301 & 104 \\
\hline & & &
\end{tabular}

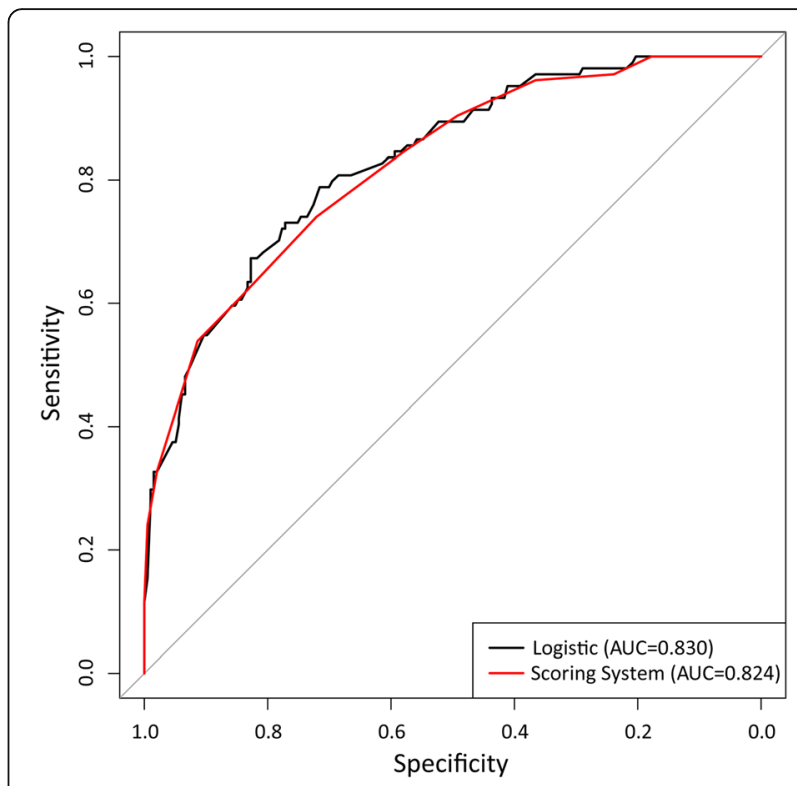

Fig. 1 Receiver operator characteristic (ROC) curves comparison between the logistic regression model and scoring system. There were no significant AUC differences between the logistic regression model and the scoring system $(p=0.239)$

calculated to be 5. Final pathology report showed that the tumor had LVI.

\section{Discussion}

In this present study, we proposed a risk-stratification score system based on MRI-derived and histopathologic features to predict LVI in breast cancer. The system incorporated six predictors including tumor margins, lobulation sign, MRI-reported ALNM, DWI appearance, TIC pattern, and HER-2 status. Our results showed that the scoring system for predicting breast LVI had good discrimination (AUC $=0.831, \quad 95 \% \mathrm{CI}$ : 0.776--0.872). Within the system, patient's risk for LVI was categorized as low ( $<3.5$ points), moderate (3.5-6.0 points) or high ( $\geq 6.0$ points).

Risk stratification scoring system has been widely created to aid in clinical decision-making and potentially improve the patients management [17-22]. To our best of knowledge, this was the first study predicted LVI in patients with breast cancer using a comprehensive risk stratification scoring system. The scoring system was developed on the basis of a logistic regression model. According to the scoring system, the predicted risk of LVI ranged from 0 to $100 \%$. For example, the risk of LVI is predicted to be $0 \%$ when a tumor had -1.5 to 2.5 points and $100 \%$ when a tumor had 7.5 or 8 points. This system provides an easy tool for clinicians to evaluate the risk of LVI in breast cancer patients prior to surgery.

We found that MRI findings including tumor margins, lobulation sign, MRI-reported ALNM, DWI appearance, 

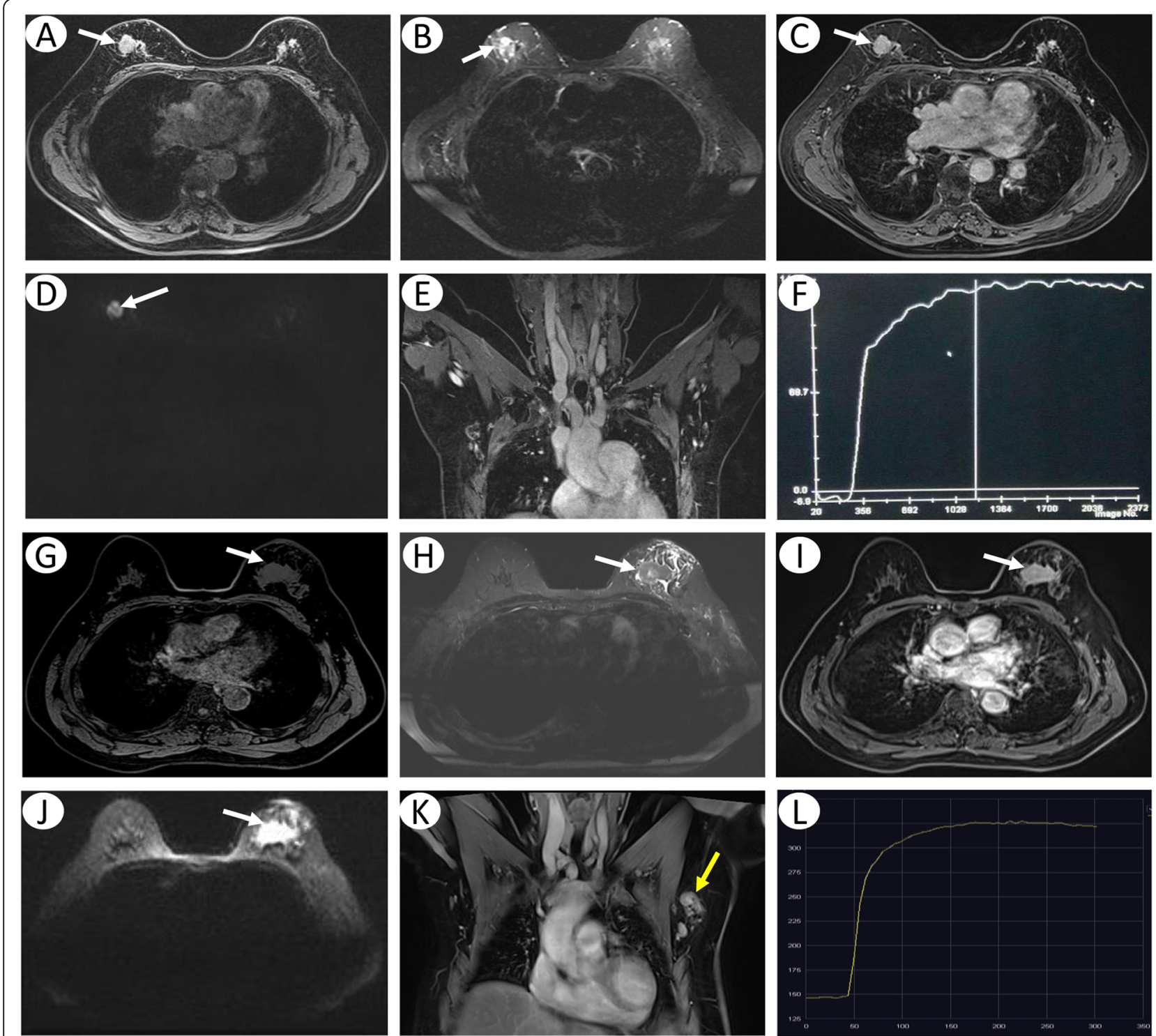

Fig. 2 Examples of the scoring system in use. MRI examinations of patient 1 including. Axial T1-weighted fat-suppression (a), T2-weighted STIR (b), axial T1-weighted fat-suppression contrast-enhancement (c), DWI (d), TIC (e) and axillary imaging (f) showed a tumor (white arrows) with maximum diameter of $19 \mathrm{~mm}$, location in the upper-outer quadrant, well-defined margins, absence of lobulation sign, obvious contrastenhancement, marked hyperintensity on DWI images, type I TIC, and no ALNM. Biopsy results showed a IDC (grade 2) and positive HER-2 (+). The risk of LVI assessed by scoring system was 1. MRI examinations of patient 2 including axial T1-weighted fat-suppression (g), T2-weighted STIR (h), axial T1-weighted fat-suppression contrast-enhancement (i), DWI (j), TIC (k) and axillary imaging (I) showed a tumor (white arrows) with maximum diameter of $35 \mathrm{~mm}$, location in the upper-inner quadrant, ill-defined margins, presence of lobulation sign, obvious contrastenhancement, marked hyperintensity on DWI images, type II TIC, and multiple ALNM (yellow arrow). Biopsy results showed a IDC (grade 3) and positive HER-2 (3+). The risk score of LVI could be calculated to be 5 (Fig. 2n). Final pathology report showed this tumor had LVI

and TIC pattern were important risk factors for the prediction of breast LVI. We found tumor with LVI usually had morphological characteristics such as illdefined margins, and lobulation sign. Tumors with irregular or spiculated or lobulated margins have a significantly higher microvessel density than tumors with smooth margins since the morphology is associated with a more aggressive behavior in breast cancer [23].
A review has shown that the presence of LVI correlates closely with locoregional lymph node involvement [24]. Although LVI was associated with MRI-reported ALNM, multiple ALNM was more frequent in tumors without LVI than those with LVI, which may due to the fact that LVI could occur before ALNM. Besides the conventional MRI, functional MRI such as DWI and DCE-MRI provides other important information about tumor diffusion and perfusion. Karan B et al. observed 
the median apparent diffusion coefficient (ADC) value was significantly associated with LVI $(P=0.008)$, which was in line with this study [25]. Malignant tumors have high cellularity but relatively less extracellular space, which has been shown to restrict water diffusion [26]. DCE-MRI has become a valuable tool to evaluate breast cancer morphologically and kinetically. Examination of the TICs allows physiological parameters related to tissue perfusion, microvascular vessel wall permeability and extravascular-extracellular volume fraction to be extracted, which may aid characterization of the underlying pathology. TIC type was the most important risk factor for LVI, the risk of LVI in tumors with TIC II and III was 10.6 and 26.0 times compared with tumors with TIC I, respectively. TIC III was more common in breast tumor with LVI. It may because TIC III is associated with peritumor inflammation. In addition, it suggests the presence of an increased vessel density and arteriovenous anastomoses with rapid outflow and thus fading of the contrast media [27]. HER-2 gene amplification or overexpression in breast cancer is a prognostic factor and predictive of a more aggressive clinical course for the patient. It is associated with high tumor-grade, hormone receptor-negative tumors, lymph node metastasis [28], increased risk of recurrence after surgery, poor response to treatment and shortened survival. This study found that the risk of LVI was significantly increased with the positive rate of HER-2 $(P<0.001)$, which was in line with a previous study performed by Ugras et al. [29].

This study also has some limitations. Firstly, we evaluated DWI appearance instead of ADC values for LVI prediction because we didn't measure ADC in routine practice. Secondly, this was a retrospective study. Thirdly, this study didn't include multifocal or multicentric carcinoma. Finally, we didn't test this scoring system in a validation cohort, which may limit the transfer of our system to other institutions.

\section{Conclusions}

In summary, our MRI and HER2 based scoring system can be applied in a preoperative setting. The system can be used to stratify LVI risk in breast cancer patients, which is helpful for clinical decision-making regarding tumor staging, treatment planning, and prognosis assessment. Patients with LVI may require for adjuvant therapies because of the high risk of recurrence and metastasis.

\footnotetext{
Abbreviations

ALVM: Axillary lymph node metastasis; AUC: Area under the curve;

Cl: Confidence interval; DCE: Dynamic contrast-enhanced; DWl: Diffusionweighted imaging; LVI: Lymphovascular invasion; MRI: Magnetic Resonance Imaging; ROC: Receiver operating characteristic curve; TIC: Time to signal intensity curve
}

\section{Acknowledgements}

None.

\section{Authors' contributions}

MYDLNJT, MHMTJDML, and GZD contributed to the conception and design of the study. MYDLNJT wrote the manuscript. DLARMAHT, ASKEJHJ,

PLDMWME, and YS contributed to collection, analysis and interpretation of data, ABDKYMJABLZ, WJS, NEAMNRZ, and MHMTJDML participated in the literature screening and data extraction. GZD and MYDLNJT reviewed and revised the manuscript. All authors read and approved the final version of the manuscript.

\section{Funding}

None.

Availability of data and materials

All data generated or analysed during this study are included in this published article.

\section{Ethics approval and consent to participate}

This study was approved by the institutional review board of the first people's Hospital of Kashi area, the need for informed patient consent for inclusion was waived.

\section{Consent for publication}

Not applicable.

\section{Competing interests}

The authors declare that they have no competing interests.

Received: 26 April 2019 Accepted: 24 January 2020

Published online: 03 February 2020

\section{References}

1. Teel P. Vascular invasion as a prognostic factor in breast carcinoma. Surg Gynecol Obstet. 1964;118:1006-8.

2. Gujam FJ, Going JJ, Edwards J, et al. The role of lymphatic and blood vessel invasion in predicting survival and methods of detection in patients with primary operable breast cancer. Crit Rev Oncol Hematol. 2014;89:231-41.

3. Paduch $R$. The role of lymphangiogenesis and angiogenesis in tumor metastasis. Cell Oncol. 2016;39:397-410.

4. Podgrabinska S, Skobe M. Role of lymphatic vasculature in regional and distant metastases. Microvasc Res. 2014;95:46-52.

5. Sleeman JP, Thiele W. Tumor metastasis and the lymphatic vasculature. Int J Cancer. 2009;125:2747-56.

6. Ouyang FS, Guo BL, Huang XY, et al. A nomogram for individual prediction of vascular invasion in primary breast cancer. Eur J Radiol. 2019;110:30-8.

7. Klingen TA, Chen Y, Stefansson IM, et al. Tumour cell invasion into blood vessels is significantly related to breast cancer subtypes and decreased survival. J Clin Pathol. 2017;70:313-9.

8. Kuhl C. Current status of breast MR imaging. Part 2. Clinical applications. Radiology. 2007;244:672-91.

9. Thomassin-Naggara I, De Bazelaire C, Chopier J, et al. Diffusionweighted MR imaging of the breast: advantages and pitfalls. Eur J Radiol. 2013;82:435-43.

10. Macchini M, Ponziani M, lamurri AP, et al. Role of DCE-MR in predicting breast cancer subtypes. Radiol Med. 2018;123(10):753-64.

11. Komatsu S, Lee CJ, Ichikawa D, et al. Predictive value of the time-intensity curves on dynamic contrast-enhanced magnetic resonance imaging for lymphatic spreading in breast cancer. Surg Today. 2005;35:720-4.

12. Fan $M, H e T$, Zhang $P$, et al. Heterogeneity of diffusion-weighted imaging in Tumours and the surrounding Stroma for prediction of Ki-67 proliferation status in breast Cancer. Sci Rep. 2017;7:2875

13. Wolff AC, Hammond ME, Hicks DG, Dowsett M, McShane LM, Allison KH, Allred DC, Bartlett JM, Bilous M, Fitzgibbons P, et al. Recommendations for human epidermal growth factor receptor 2 testing in breast cancer: American Society of Clinical Oncology/College of American Pathologists clinical practice guideline update. J Clin Oncol. 2013;31:3997-4013.

14. Pathmanathan N, Balleine RL. Ki67 and proliferation in breast cancer. J Clin Pathol. 2013;66:512-6.

15. Pinder S, Ellis IO, Galea M, et al. Pathological prognostic factors in breast cancer. III. Vascular invasion: relationship with recurrence and survival in a large study with long-term follow-up. Histopathology. 1994;24:41-7. 
16. Sullivan LM, Massaro JM, D'Agostino RS. Presentation of multivariate data for clinical use: the Framingham study risk score functions. Stat Med. 2004;23: $1631-60$.

17. Hustedt JW, Chung A, Bohl DD. Development of a Risk Stratification Scoring System to Predict General Surgical Complications in Hand Surgery Patients. J Hand Surg Am. 2018:43:641-648.e6.

18. González-Tablas M, Crespo I, Vital AL, et al. Prognostic stratification of adult primary glioblastoma multiforme patients based on their tumor gene amplification profiles. Oncotarget. 2018;9:28083-102.

19. Hong MJ, Na DG, Baek JH, et al. Cytology-ultrasonography risk-stratification scoring system based on fine-needle aspiration cytology and the Koreanthyroid imaging reporting and data system. Thyroid. 2017;27:953-9.

20. Huang $\mathrm{DD}$, Zhuang $\mathrm{CL}$, Wang $\mathrm{SL}$, et al. Prediction of prolonged postoperative ileus after radical Gastrectomy for gastric Cancer: a scoring system obtained from a prospective study. Medicine (Baltimore). 2015:94: e2242.

21. Yu YB, Gau JP, Liu CY, et al. A nation-wide analysis of venous thromboembolism in 497,180 cancer patients with the development and validation of a risk-stratification scoring system. Thromb Haemost. 2012;108: 225-35.

22. Kawachi Y, Nakashima A, Toshima Y, et al. Evaluation of the quality of cardiovascular surgery care using risk stratification analysis according to the EuroSCORE additive model. Circ J. 2002;66:145-8.

23. Fernández-Guinea O, Andicoechea A, González LO, et al. Relationship between morphological features and kinetic patterns of enhancement of the dynamic breast magnetic resonance imaging and clinico-pathological and biological factors in invasive breast cancer. BMC Cancer. 2010;10:8.

24. Rampaul RS, Pinder SE, Elston CW, et al. Prognostic and predictive factors in primary breast cancer and their role in patient management: the Nottingham breast team. Eur J Surg Oncol. 2001;27:229-38.

25. Karan B, Pourbagher A, Torun N. Diffusion-weighted imaging and ${ }^{18} \mathrm{~F}$ fluorodeoxyglucose positron emission tomography/computed tomography in breast cancer: correlation of the apparent diffusion coefficient and maximum standardized uptake values with prognostic factors. J Magn Reson Imaging. 2016:43:1434-44.

26. Bonekamp S, Corona-Villalobos CP, Kamel IR. Oncologic applications of diffusion-weighted MRI in the body. J Magn Reson Imaging. 2012;35: 257-79.

27. Frouge C, Guinebretiere JM, Contesso G, et al. Correlation between contrast enhancement in dynamic magnetic resonance imaging of the breast and tumor angiogenesis. Investig Radiol. 1994;29:1043-9.

28. Eccles SA. The role of c-erbB-2/HER2/neu in breast cancer progression and metastasis. J Mammary Gland Biol Neoplasia. 2001;6:393-406.

29. Ugras S, Stempel M, Patil S, et al. Estrogen receptor, progesterone receptor, and HER2 status predict lymphovascular invasion and lymph node involvement. Ann Surg Oncol. 2014;21:3780-6.

\section{Publisher's Note}

Springer Nature remains neutral with regard to jurisdictional claims in published maps and institutional affiliations.

Ready to submit your research? Choose BMC and benefit from:

- fast, convenient online submission

- thorough peer review by experienced researchers in your field

- rapid publication on acceptance

- support for research data, including large and complex data types

- gold Open Access which fosters wider collaboration and increased citations

- maximum visibility for your research: over $100 \mathrm{M}$ website views per year

At $\mathrm{BMC}$, research is always in progress.

Learn more biomedcentral.com/submissions 